\title{
Ocular pulse amplitude in different types of glaucoma using dynamic contour tonometry: Diagnosis and follow-up of glaucoma
}

\author{
LINGYAN CHENG $^{1 *}$, YUZHI DING ${ }^{2 *}$, XUANCHU DUAN ${ }^{3}$ and ZHIFENG WU ${ }^{1}$ \\ ${ }^{1}$ Department of Ophthalmology, Wuxi Second People's Hospital of Nanjing Medical University, \\ Wuxi, Jiangsu 214000; ${ }^{2}$ Department of Ophthalmology, Zhongda Hospital, Medical School of \\ Southeast University, Nanjing, Jiangsu 210009; ${ }^{3}$ Department of Ophthalmology, Xiangya No. 2 \\ Hospital Affiliated to Central South University, Changsha, Hunan 410008, P.R. China
}

Received May 12, 2016; Accepted March 24, 2017

DOI: $10.3892 / e t m .2017 .5074$

\begin{abstract}
The aim of the present study was to compare the ocular pulse amplitude (OPA) in patients with different types of glaucoma using dynamic contour tonometry (DCT), to evaluate ocular and systemic factors associated with the OPA and to verify whether OPA measured by DCT is an independent predictor for glaucoma diagnosis. A total of 217 eyes of 217 participants in the following five groups were included in this cross-sectional study: Chronic angle closure glaucoma (CACG), primary open angle glaucoma, normal tension glaucoma (NTG), suspected open angle glaucoma (SOAG) and normal control (NC). The following tests were simultaneously performed during a single visit: Intra-ocular pressure (IOP), OPA, cup-to-disk (C/D) ratio, mean damage (MD) and loss variance (LV). OPAs were compared in each group. The association between OPA and IOP, age, $\mathrm{C} / \mathrm{D}$ ratio, $\mathrm{MD}$ and $\mathrm{LV}$ was detected. OPA analysis prior to and after trabeculectomy was also performed to assess its prognostic value. Among the 217 individuals, the OPA was consistent with the IOP, both measured by DCT, along with the MD and LV. Patients with CACG and SOAG had higher OPA values than those with NTG and normal controls. Compared with patients aged $>30$ years, the OPA was significantly lower in younger patients, while they may not have been affected by different $C / D$ ratios. After trabeculectomy, the OPA had significantly decreased compared with the values prior to surgery. In conclusion, the present study showed that the OPA is correlated with the IOP determined by DCT. CACG and SOAG patients had higher OPA values than patients with other types of glaucoma. OPA
\end{abstract}

Correspondence to: Professor Zhifeng Wu, Department of Ophthalmology, Wuxi Second People's Hospital of Nanjing Medical University, 68 Zhongshan Road, Wuxi, Jiangsu 214000, P.R. China E-mail: 569562916@qq.com

${ }^{*}$ Contributed equally

Key words: ocular pulse amplitude, dynamic contour tonometry, intraocular pressure, glaucoma measured by DCT may be a predictor for glaucoma diagnosis and prognosis.

\section{Introduction}

In patients with glaucoma, the diagnosis and decision making regarding treatment modalities require precise assessment of the intraocular pressure (IOP) $(1,2)$. At present, Goldmann applanation tonometry (GAT) is a widely accepted international gold standard for the measurement of the $\operatorname{IOP}(3,4)$. However, in eyes with abnormal central corneal thickness (CCT) and structural corneal rigidity, the accuracy of this method has been questioned (5). Dynamic contour tonometry (DCT) may be an ideal alternative means of measuring the IOP, as the tonometer has a wide safety range of appositional force that reduces operator bias and is not affected by the tear film or a force to deform the cornea $(6,7)$. The physical principle of DCT is the following: The pressure measured at the surface of the eye equals the pressure inside the eye when the contours of the corneal surface and the tonometer match $(8,9)$.

In addition to IOP measurement, this tonometer is also capable of measuring the ocular pulse amplitude (OPA), which is defined as the difference between the minimum (or diastolic) and maximum (or systolic) values of the pulsatile IOP. The importance of the OPA in a clinical glaucoma setting remains to be established, and studies on the OPA in different types of glaucoma and controls are required.

The present study compared the OPA and IOP in patients with chronic angle closure glaucoma (CACG), primary open angle glaucoma (POAG), normal tension glaucoma (NTG) and suspected open angle glaucoma (SOAG) as well as normal controls (NC) using DCT. The OPA among these patients groups was compared and its correlation with IOP and other factors associated with glaucoma was assessed.

\section{Patients and methods}

Subjects. A total of 127 consecutive patients (63 males and 64 females) with different types of glaucoma were included in the study with the age ranging from 12 to 89 years (mean age, $54.56 \pm 17.84$ years). The mean age of the 90 healthy volunteers 
(42 males and 48 females) was $41.23 \pm 17.62$ years, ranging from 10 to 76 years old. All of them provided informed patient consent prior to the study and the study protocol was approved by the Ethics Committee of Wuxi Second People's Hospital, Nanjing, China. A total of 30 patients (30 eyes) with different types of glaucoma who were to receive trabeculectomy for the first time were included. Each patient and healthy subject was individually assessed by DCT. Eyes were included in the study if they met the following inclusion criteria: Group 1 (CACG group); diagnosis of CACG based on elevated intraocular pressure $\geq 21 \mathrm{mmHg}$, progressing angle closure and damage of optic nerve, closed angle on gonioscopy, coherency between peripheral iris and meshwork, typical glaucomatous visual field defects and cupping of the optic disk without acute increase of IOP. Group 2 (POAG group); diagnosis of POAG based on elevated intraocular pressure $>21 \mathrm{mmHg}$ (based on an average of there IOP readings on GAT), open angle on gonioscopy, glaucomatous visual field defects, glaucomatous cupping of the optic disk and defects of retinal nerve fiber layer. Group 3 (NTG group); diagnosis of NTG based on normal intraocular pressure $(\leq 21 \mathrm{mmHg})$, open angle on gonioscopy, glaucomatous visual field defects, glaucomatous cupping of the optic disk and defects of retinal nerve fiber layer. Diseases leading to optic nerve damage such as intracal occupying lesion were ideally excluded using computed tomography or magnetic resonance imaging. Group 4 (SOAG group); diagnosis of SOAG based on elevated intraocular pressure $(\geq 21 \mathrm{mmHg}$ ) during several IOP measurements at 24-hour intervals, large optic cup, significant asymmetry of optic cup ( $>0.2$ ) between the two eyes. Group 5 (NC group, healthy volunteers); normal intraocular pressure $(\leq 21 \mathrm{mmHg})$ with no family history of glaucoma, $\mathrm{C} / \mathrm{D} \leq 0.3$, normal limbal anterior chamber depth ( $\geq 1 / 3$ cornea thickness), with the exception of mild cataract in the old and ametropia, no other eye diseases were observed.

Patients were excluded from the study if they had the following: i) Corneal diseases such as corneal macula and corneal edema; ii) iridocyclitis; iii) severe myopia; iv) lens opacity; v) poor performance in stare test; vi) use of corticosteroid hormones; vii) history of intraocular surgery or ocular trauma; viii) recent wearing of corneal contact lenses; ix) previous filtering/drainage procedure or any other intraocular surgery.

History of medication use was documented and considered in the analysis. The total number of topical IOP-lowering medications and the frequency of their use were noted.

Assessments. Patients were studied prospectively, with visual examinations and tests performed in the following sequence during their visit: IOP and OPA measurements using DCT (Pascal DCT, SMT Swiss Microtechnology AG, Port, Switzerland), and visual field tests using oculus twinfield perimetry. Subjects were examined in a random order and each tonometer was used three times separately, with a 15-minute interval between each measurement. Each patient undergoing trabeculectomy was subjected to DCT measurement separately one day prior to surgery and at one week post-operation. All of the measurements were performed by the same examiner. As DCT offers a reliability coefficient $(\mathrm{Q}=1-5)$ for each reading, it indicates a poorer reliability if a higher Q-value is obtained. In the present study, only those readings with $\mathrm{Q} \leq 3$ were used. GAT measurements were performed on a slit lamp with a tonometer calibrated according to the manufacturer's instructions under topical anesthesia. Three GAT readings were obtained for each eye, with a 5-min interval, and mean IOP was recorded. MD and LV was detected by Oculus Twinfield perimetry (Oculus Optikgeraete GmbH, Wetzlar, Germany) and C/D ratio was provided by Cirrus HD-OCT 4000 (Carl Zeiss AG, Oberkochen, Germany).

Statistical analysis. Values are expressed as the mean \pm standard deviation. During the evaluation of the study data, along with the descriptive statistical methods, parameters with normal distribution for the comparison of qualitative data were evaluated using a paired-samples t-test. Pearson's correlation coefficients were used to assess the correlation between OPA and other parameters. $\mathrm{P}<0.05$ was considered to indicate a statistically significant difference. Statistical calculations were performed using SPSS 11.5 (SPSS, Inc., Chicago, IL, USA).

\section{Results}

Population. A total of 217 eyes of 217 glaucoma patients were included in the present study. The mean age was $54.56 \pm 17.84$ (range, 12-89) years and the patient cohort included 63 males and 64 females. The patients were divided into the following five groups: CACG $(n=37)$, POAG $(n=35), N T G(n=20)$, SOAG $(n=35)$ and $\mathrm{NC}(\mathrm{n}=90)$. The mean cup-to-disk $(\mathrm{C} / \mathrm{D})$ ratio was $0.738 \pm 0.22$ (range, $0.3-1$ ). The OPA value varied from 0.7 to $8.9 \mathrm{mmHg}$, with a mean of $2.85 \pm 1.41 \mathrm{mmHg}$. Table I summarizes the patient characteristics in the different diagnostic groups.

Correlation between OPA and IOP. The OPA was found to be significantly correlated with the IOP measured by DCT, and the correlation coefficient was 0.503 ( $\mathrm{P}<0.001$; Fig. 1A). There was a significant positive association between OPA and IOP (slope estimate, 0.159; $\mathrm{P}<0.001$ ), indicating that the OPA increased by $\sim 1.6 \mathrm{mmHg}$ with every $10-\mathrm{mmHg}$ elevation in IOP measured by DCT. Fig. 1B directly shows the mean OPA values in each group. After comparing OPA among different types of glaucoma and normal controls (Table II), it was found that the average OPA values were highest in the CACG group $(3.17 \pm 1.76 \mathrm{mmHg})$. The NTG group had the lowest OPA values $(2.28 \pm 0.53 \mathrm{mmHg})$. The CACG group had significantly higher OPA values than the NTG $(\mathrm{P}=0.014)$ and normal control group $(\mathrm{P}=0.018)$. There was a similar significance of the difference of the SOAG group from the NTG and normal control group ( $\mathrm{P}=0.028$ and 0.044 , respectively). Table II also shows the mean IOP values detected by DCT as well as GAT in each of these groups. The results indicated that DCT IOP values were in good correspondence with the GAT IOP, with a correlation coefficient of $0.867(\mathrm{P}<0.001$, Fig. 2).

Correlation between OPA and visual field parameters. The correlation of the OPA with the mean damage (MD) and loss variance $(\mathrm{LV})$ on the oculus twinfield perimetry was assessed 
Table I. Patient characteristics.

\begin{tabular}{lrccc}
\hline Group & $\mathrm{N}$ & $\begin{array}{c}\text { Mean age, } \\
\text { years (range) }\end{array}$ & $\begin{array}{c}\text { Males vs. } \\
\text { females (n) }\end{array}$ & $\begin{array}{c}\text { Mean C/D } \\
\text { ratio (range) }\end{array}$ \\
\hline Total & 127 & $54.56 \pm 17.84$ & $63 / 64$ & $0.738 \pm 0.22$ \\
SOAG & 35 & $51.43 \pm 16.50$ & $15 / 20$ & $0.698 \pm 0.20$ \\
CACG & 37 & $62.43 \pm 9.14$ & $13 / 24$ & $0.664 \pm 0.25$ \\
POAG & 35 & $44.63 \pm 20.70$ & $20 / 15$ & $0.781 \pm 0.20$ \\
NTG & 20 & $62.20 \pm 17.79$ & $15 / 5$ & $0.855 \pm 0.11$ \\
NC & 90 & $41.23 \pm 17.62$ & $42 / 48$ & $0.267 \pm 0.28$ \\
\hline
\end{tabular}

SOAG, suspected open angle glaucoma; CACG, chronic angle closure glaucoma; POAG, primary open angle glaucoma; NTG, normal tension glaucoma; $\mathrm{C} / \mathrm{D}$ ratio, cup-to-disk ratio.

Table II. GAT IOP, DCT IOP and DCT OPA in each of the groups.

\begin{tabular}{lcrcc}
\hline Group & $\mathrm{N}$ & $\begin{array}{c}\text { GAT IOP } \\
(\mathrm{mmHg})\end{array}$ & $\begin{array}{c}\text { DCT IOP } \\
(\mathrm{mmHg})\end{array}$ & $\begin{array}{c}\text { DCT OPA } \\
(\mathrm{mmHg})\end{array}$ \\
\hline $\begin{array}{l}\text { Total } \\
\text { glaucoma }\end{array}$ & 127 & $16.67 \pm 5.22$ & $18.45 \pm 4.91$ & $2.85 \pm 1.41$ \\
SOAG & 35 & $17.86 \pm 2.95$ & $19.47 \pm 2.81$ & $3.09 \pm 1.25^{\mathrm{a}, \mathrm{b}}$ \\
CACG & 37 & $15.16 \pm 4.92$ & $16.94 \pm 4.21$ & $3.17 \pm 1.76^{\mathrm{a}, \mathrm{b}}$ \\
POAG & 35 & $18.2 \pm 7.40$ & $20.20 \pm 7.10$ & $2.60 \pm 1.39$ \\
NTG & 20 & $14.7 \pm 2.43$ & $16.39 \pm 2.36$ & $2.28 \pm 0.53$ \\
NC & 90 & $14.27 \pm 2.81$ & $17.33 \pm 2.71$ & $2.56 \pm 1.18$ \\
\hline
\end{tabular}

Values are expressed as the mean \pm standard deviation. ${ }^{\mathrm{a}} \mathrm{P}<0.05$ vs NTG group. ${ }^{b} \mathrm{P}<0.05$ vs. NC group. GAT IOP, intraocular pressure measured by Goldmann applanation tonometry; DCT OPA, ocular pulse amplitude determined by dynamic contour tonometry.

in order to determine whether visual field severity has any correlation with the OPA. It was found that the OPA correlates well with $\mathrm{MD}$ and $\mathrm{LV}$, with the correlation coefficients being $0.322(\mathrm{P}=0.02)$ and $0.354(\mathrm{P}=0.01)$, respectively (Fig. 3).

Association between OPA and age. In order to determine whether OPA varies in different age groups, patients were divided into the following three age groups: Group $1,<31$ years; Group 2, 31-60 years; Group 3, >60 years. The OPA values in Group 1 were significantly lower than those in Groups 2 and $3(\mathrm{P}=0.022$ and 0.025 , respectively). There was no significant difference between Groups 2 and $3(\mathrm{P}=0.915$; Fig. 4A).

Association between OPA and surgery. Comparison of the OPA prior to and subsequent to surgery revealed that OPA values obviously decreased by $1.89 \pm 1.76 \mathrm{mmHg}$ after surgery $(\mathrm{P}<0.05$; Fig. 4B). Twenty-eight patients who had a decreased OPA following surgery formed perfect blebs which possessed a fine filtered function one week after surgery, while the other two patients who had an increased OPA were found to have an
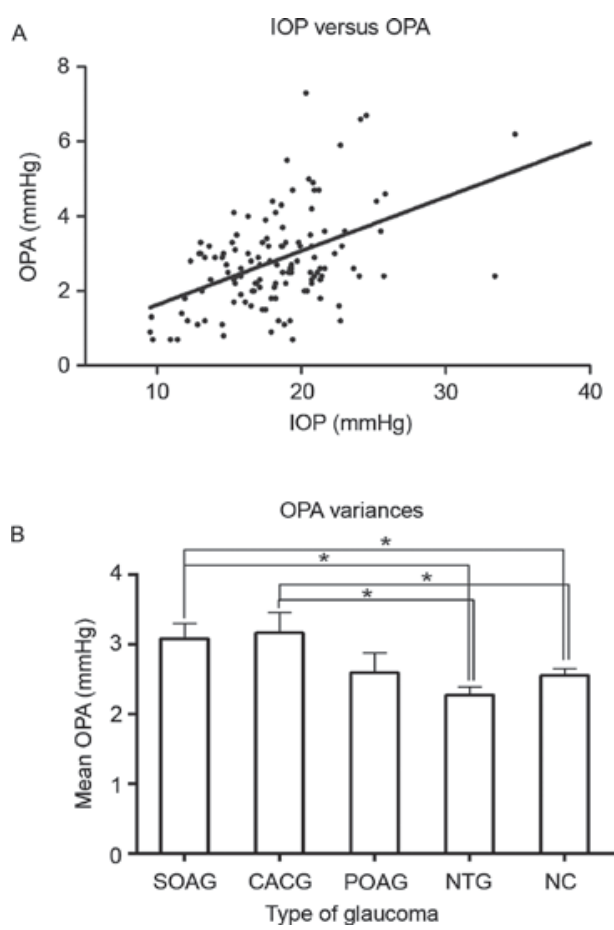

Figure 1. (A) Correlation between IOP and OPA measured by dynamic contour tonometry (correlation coefficient, $0.503 ; \mathrm{P}<0.001$ ). (B) OPA among different types of glaucoma. ${ }^{*}<<0.05$. Groups: SOAG, suspected open angle glaucoma $(\mathrm{n}=35) ;$ CACG, chronic angle closure glaucoma $(\mathrm{n}=37)$; POAG, primary open angle glaucoma $(n=35)$; NTG, normal tension glaucoma $(n=20)$; NC, normal controls $(n=90)$. OPA, ocular pulse amplitude; IOP, intraocular pressure.

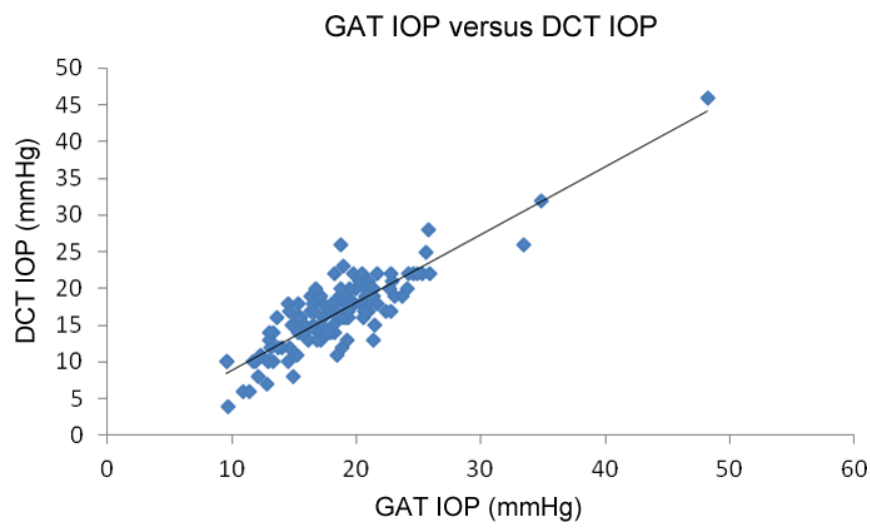

Figure 2. Correlation between GAT IOP and DCT IOP. Correlation coefficient, 0.867; $\mathrm{P}<0.001$. IOP, intraocular pressure; GAT, Goldmann applanation tonometry; DCT, dynamic contour tonometry.

increased IOP as well; in these patients, the IOP was difficult to control and they had a congestive and flat bleb.

\section{Discussion}

DCT is a novel principle of IOP measurement that is totally independent of central corneal thickness, thus presumed to provide more precise IOP measurements than other methods. The tonometer is capable of measuring the OPA, which represents the difference between systolic and diastolic values of the IOP. It may be compared with the pulse pressure, which is the difference between systolic and diastolic values of systemic 

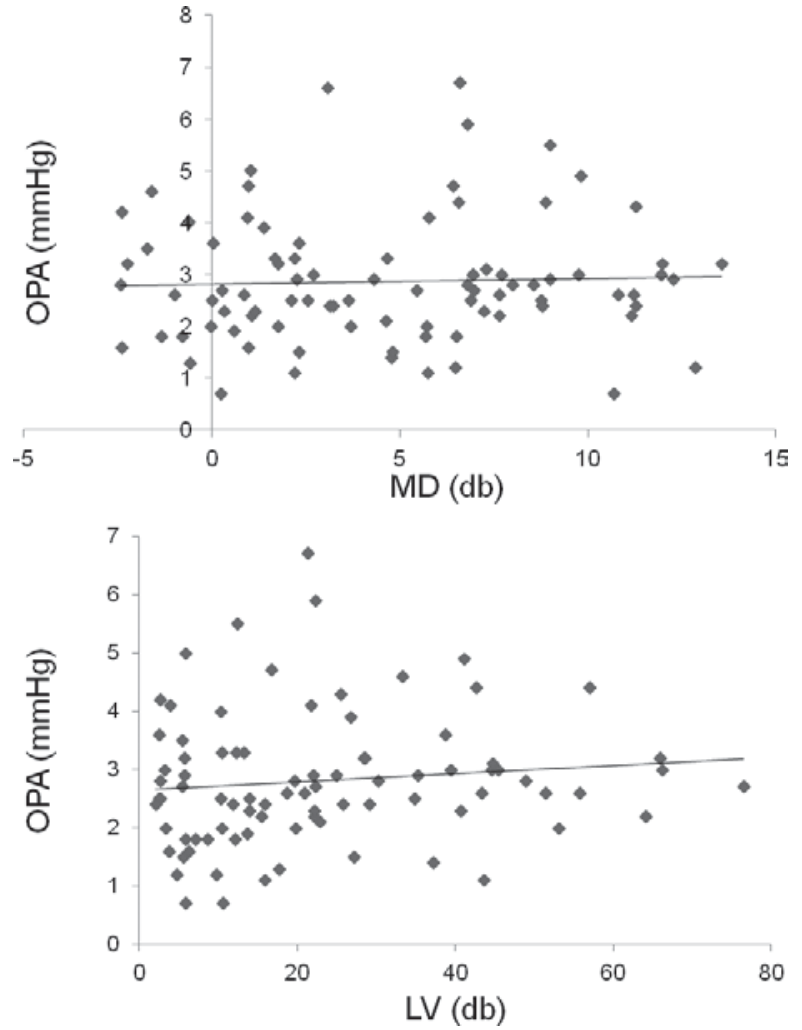

Figure 3. Correlation of MD (correlation coefficient, 0.322; $\mathrm{P}=0.02$ ) and LV (correlation coefficient, $0.354 ; \mathrm{P}=0.01$ ) with $\mathrm{OPA}$. MD, mean damage; $\mathrm{LV}$, loss variance; OPA, ocular pulse amplitude.
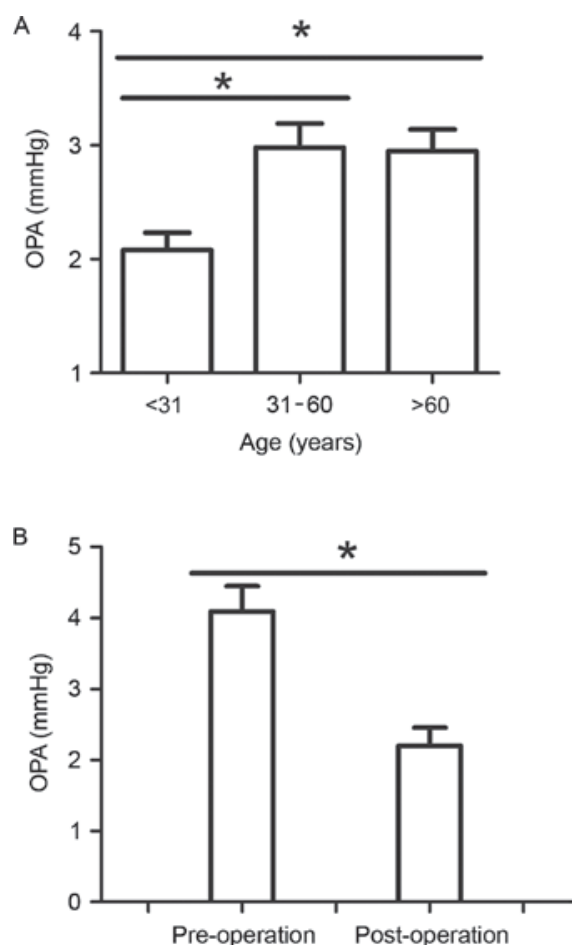

Figure 4. Effect of (A) patient age and (B) surgery on OPA determined by dynamic contour tonometry. ${ }^{*} \mathrm{P}<0.05$. OPA, ocular pulse amplitude.

blood pressure (10). OPA is considered to be the objective parameter reflecting choroidal perfusion and fluctuation of the IOP, thus having significance in the clinical evaluation of
IOP constancy. The OPA may also be used to detect severe cardiovascular disease as a non-invasive and affordable screening tool (11). The present study identified that the DCT OPA measurements are in good concordance with the DCT IOP. Measured by DCT, the OPA values were consistent with the IOP values.

This positive correlation between OPA and IOP has been previously reported in healthy and glaucomatous eyes (8,12-14). Age may have an important influence on DCT OPA. Patients with lower average age $(<31$ years) showed a significantly lower OPA value. While there was no linear correlation between OPA and age of glaucoma patients, those aged $<31$ years indeed had a significantly lower OPA than the others, which may be due to better eyeball resilience and lower outflow resistance of aqueous fluid. C/D ratios are likely to have no influence on OPA, which is similar to the finding of a study by Romppainen et al (15). In fact, scleral properties (rigidity) may vary with the level of IOP, which may contribute to the fact that OPA increases with higher IOP.

The present study evaluated the differences in DCT OPA among CACG, SOAG, POAG, NTG patients as well as healthy controls. The DCT OPA in the CACG and SOAG groups was significantly higher than that in the NTG and normal control groups, while that in the POAG group was not significantly different from that in the other groups. It has been previously reported that the OPA in NTG patients was significantly lower $(16,17)$, while that in POAG patients was significantly higher than that in healthy controls (18-20). In the present study, the OPA in the POAG group was higher than that in the NTG group, while this difference was not statistically significant, probably due to the small sample size in the NTG group. However, detection of the OPA may still be promising for clinical diagnosis: Owing to the high value in the CACG group, the OPA may be supportive in the diagnosis of CACG patients who have no symptoms or typical glaucomatous visual field damage in the early stage.

Another interesting observation in the present study was that the OPA value was obviously increased with the MD and $\mathrm{LV}$ parameters of the visual field. This result is in accordance with the findings from Vulsteke et al (13), indicating that, besides the progression of visual field loss, an increased OPA value may be a reliable indicator of the severity of glaucoma; more importantly, the OPA value is not influenced by subjective factors. Therefore, the OPA may be used as a significant indicator giving direction for decisions regarding the treatment of glaucoma.

The present study found that $93.3 \%$ of glaucoma patients had reduced OPA values after trabeculectomy and $92.9 \%$ of them formed perfect blebs with a good filtering function at one week after the operation. Such changes in the OPA appear to illustrate that the OPA value is dependent on intraocular perfusion pressure, which is altered by trabeculectomy. In addition, high post-operative OPA values reflect the high amplitude of the pulsatile ocular tension (21), which may accelerate the formation of fibrillation and scar, which unavoidably induce the failure of surgery. Therefore, the OPA value represents a macroscopic parameter that predicts the bleb condition and prognosis at an early stage after trabeculectomy in those patients who require frequent follow-up and possibly additional intervention. Patients who have decreased OPA values 
are likely to form perfect blebs which possesses fine filtered function one week after surgery, while those who had increased OPA values were found to have increased IOP values as well, such patients' intraocular pressure are always difficult to be controlled and they are more likely to form congestive and flat bleb. Our study indicated that OPA values measured by DCT can be a predictor for glaucoma diagnosis and prognosis.

\section{Acknowledgements}

The authors would like to thank Dr Yinong Zhang (Department of Ophthalmology, Wuxi Second People's Hospital of Nanjing Medical University, Nanjing, China) for the help in contacting the participants and caring for them. Colleagues who contributed to the recording and analysis of the data are also acknowledged.

\section{References}

1. Brown KE and Congdon NG: Corneal structure and biomechanics: Impact on the diagnosis and management of glaucoma. Curr Opin Ophthalmol 17: 338-343, 2006.

2. Punjabi OS, Kniestedt C, Stamper RL and Lin SC: Dynamic contour tonometry: Principle and use. Clin Exp Ophthalmol 34: 837-840, 2006

3. Troost R, Vogel A, Beck S, Schwenn O, Grus F and Pfeiffer N: Clinical comparison of two intraocular pressure measurement methods: SmartLens dynamic observing tonography versus Goldmann. Graefes Arch Clin Exp Ophthalmol 239: 889-892, 2001.

4. Whitacre MM, Stein RA and Hassanein K: The effect of corneal thickness on applanation tonometry. Am J Ophthalmol 115: 592-596, 1993

5. Brandt JD: Corneal thickness in glaucoma screening, diagnosis, and management. Curr Opin Ophthalmol 15: 85-89, 2004.

6. Chihara E: Assessment of true intraocular pressure: The gap between theory and practical data. Surv Ophthalmol 53: 203-218, 2008.

7. Jonas $\mathrm{J}$ and Dichtl A: Optic disc morphology in myopic primary-open angle glaucoma. Graefes Arch Clin Exp Ophthalmol 235: 627-633, 1997.

8. Punjabi OS, Ho HK, Kniestedt C, Bostrom AG, Stamper RL and Lin SC: Intraocular pressure and ocular pulse amplitude comparisons in different types of glaucoma using dynamic contour tonometry. Curr Eye Res 31:851-862, 2006.
9. Knecht PB, Bosch MM, Michels S, Mannhardt S, Schmid U, Bosch MA and Menke MN: The ocular pulse amplitude at different intraocular pressure: A prospective study. Acta Ophthalmol 89: e466-e471, 2011

10. Pourjavan S, Boëlle PY, Detry-Morel M and De Potter P. Physiological diurnal variability and characteristics of the ocular pulse amplitude (OPA) with the dynamic contour tonometer (DCT-Pascal). Int Ophthalmol 27: 357-360, 2007.

11. Bertelmann T, Langanke S, Potstawa M and Strempel I: Can dynamic contour tonometry and ocular pulse amplitude help to detect severe cardiovascular pathologies? Clin Ophthalmol 8: 1317-1321, 2014.

12. Stalmans I, Harris A, Vanbellinghen V, Zeyen T and Siesky B: Ocular pulse amplitude in normal tension and primary open angle glaucoma. J Glaucoma 17: 403-407, 2008.

13. Vulsteke C, Stalmans I, Fieuws S and Zeyen T: Correlation between ocular pulse amplitude measured by dynamic contour tonometer and visual field defects. Graefes Arch Clin Exp Ophthalmol 246: 559-565, 2008.

14. Kniestedt C, Lin S, Choe J, Nee M, Bostrom A, Stürmer J and Stamper RL: Correlation between intraocular pressure, central corneal thickness, stage of glaucoma, and demographic patient data: Prospective analysis of biophysical parameters in tertiary glaucoma practice populations. J Glaucoma 15: 91-97, 2006.

15. Romppainen T, Kniestedt C, Bachmann LM and Stürmer J: Ocular pulse amplitude: A new biometrical parameter for the diagnose of glaucoma? Ophthalmologe 104: 230-235, 2007 (In German).

16. Schmidt KG, Rückmann AV, Mittag TW, Hessemer V and Pillunat LE: Reduced ocular pulse amplitude in low tension glaucoma is independent of vasospasm. Eye (Lond) 11: 485-488, 1997.

17. Schmidt KG, von Rückmann A, Geyer O and Mittag TW: Effect of nifedipine on ocular pulse amplitude in normal pressure glaucoma. Klin Monbl Augenheilkd 210: 355-359, 1997 (In German).

18. Mittag TW, Serle J, Schumer R, Brodie S, Stegman D, Schmidt KG, Taniguchi T, Rho SH and Podos S: Studies of the ocular pulse in primates. Surv Ophthalmol 38: S183-S190, 1994.

19. Schmidt KG, von Rückmann A and Mittag TW: Ocular pulse amplitude in ocular hypertension and open-angle glaucoma. Ophthalmologica 212: 5-10, 1998 (In German).

20. Choi J, Lee J, Park SB, Lee KS, Sung KR and Kook MS: Factors affecting ocular pulse amplitude in eyes with open angle glaucoma and glaucoma suspect eyes. Acta Ophthalmol 90: 552-558, 2012.

21. von Schulthess SR, Kaufmann C, Bachmann LM, Yanar A and Thiel MA: Ocular pulse amplitude after trobeculectomy. Graefes Arch Clin Exp Ophthalmol 244: 46-51, 2006. 